\title{
ARTIGO 20
}

\section{O MÉTODO PARA CONSTRUÇÃO DE APLICATIVO-GUIA NO MESTRADO PROFISSIONALEM ENFERMAGEM}

Vanda Aparecida Tolari ${ }^{1}$, Márcia Helena de Souza Freire ${ }^{2}$

Objetivo: Descrever o método de desenvolvimento de uma tecnologia educacional, na modalidade de aplicativo-guia, para o manejo do potencial doador pediátrico, realizada como produto do Mestrado Profissional. Metodologia: relato de um método científico aplicado. Utilizou-se os passos propostos por Echer, com a perspectiva de apresentar o percurso para a produção da Tecnologia Educacional. Resultados: A construção está sustentada na Teoria das Necessidades Humanas Básicas, nos Diagnósticos e Intervenções de Enfermagem. Foi realizado mapeamento cruzado, dos indicadores selecionados, a partir das necessidades humanas básicas, decorrentes das alterações fisiopatológicas e as percebidas na avaliação clínica, com os Diagnósticos de Enfermagem, segundo taxonomia de NANDA-I e Intervenções de enfermagem baseadas nos diagnósticos, sob a ótica da manutenção dos órgãos a serem doados. Conclusão: O Aplicativo-guia subsidiará o Enfermeiro no planejamento da assistência de enfermagem segura e propiciará um impacto social no processo de transplantes em pediatria.

Descritores: Morte Encefálica, Métodos, Tecnologia Educacional, Processo de Enfermagem.

\section{THE METHOD FOR BUILDING GUIDE APPLICATIONS IN THE NURSING PROFESSIONAL MASTER}

Objective: To describe the method of development of an educational technology as a guide application for the management of potential pediatric donors, carried out as a product of the Professional Master. Methodology: report of an applied scientific method. The steps proposed by Echer5 were used, with the perspective of presenting the path for the production of Educational Technology. Results: It is supported by the Theory of Basic Human Needs, Nursing Diagnoses and Interventions. Cross-mapping of selected indicators was performed based on basic human needs, resulting from pathophysiological changes and those perceived in clinical evaluation, with Nursing Diagnoses, according to NANDA-I taxonomy and Nursing interventions based on nursing diagnoses, under the optics of the maintenance of the organs to be donated. Conclusion: The Guide application will support the Nurse in the planning of safe nursing care and will provide a social impact on the process of transplantation in pediatrics.

Descriptors: Brain Death, Methods, Educational Technology, Nursing Process.

\section{EL MÉTODO PARA LAAPLICACIÓN DE LA GUÍA DE CONSTRUCCIÓN EN EL MAESTRO PROFESIONAL DE ENFERMERÍA}

Objetivo: Describir el método de desarrollo de una tecnología educativa como una aplicación de guía para el manejo de posibles donantes pediátricos, realizada como producto del Master Profesional. Metodología: informe de un método científico aplicado. Se utilizaron los pasos propuestos por Echer5, con la perspectiva de presentar el camino para la producción de Tecnología Educativa. Resultados: está respaldado por la Teoría de las necesidades humanas básicas, los diagnósticos e intervenciones de enfermería. El mapeo cruzado de los indicadores seleccionados se realizó con base en las necesidades humanas básicas, como resultado de los cambios fisiopatológicos y los percibidos en la evaluación clínica, con los Diagnósticos de Enfermería, de acuerdo con la taxonomía NANDA-I y las intervenciones de Enfermería basadas en diagnósticos de enfermería, bajo La óptica del mantenimiento de los órganos a donar. Conclusión: La aplicación de la Guía apoyará a la Enfermera en la planificación de la atención de enfermería segura y proporcionará un impacto social en el proceso de trasplante en pediatría.

Descriptores: Muerte Cerebral, Métodos, Tecnología Educativa, Proceso de Enfermería. 


\section{INTRODUÇÃO}

No processo de vida e morte, que é permeado por significados sociais e culturais, está inserida a temática da Morte Encefálica, a qual apresenta entre os países diferentes conceitos e abordagens. No Brasil, a Resolução no 2173/2017, define que "A morte encefálica (ME) é estabelecida pela perda definitiva e irreversivel das funções do encéfalo por causa conhecida, comprovada e capaz de provocar o quadro clínico"(1). Normativa que orienta os critérios para abertura do Protocolo de Diagnóstico da ME.

AME prevêváriasalterações fisiopatológicas compotencial para a inviabilização dos órgãos e tecidos para doação ${ }^{(2)}$. A assistência de enfermagem na suspeita de ME em se tratando de criança, se configura como um processo complexo pois, requer do enfermeiro conhecimento das ciências básicas, da normativa atual, do protocolo estabelecido em seu cenário de prática, para além de entender o processo da ME e, as alterações decorrentes do mesmo.

Nesta perspectiva, o profissional enfermeiro é responsável por traçar um Plano de Cuidados que seja seguro, composto por ações de prevenção e manejo das alterações e também, por compartilhá-lo com a equipe de trabalho, tanto com a de enfermagem como a multiprofissional. Delineamento este indispensável para a manutenção do potencial de doação.

O Conselho Federal de Enfermagem - COFEN, na Resolução no 358, de 2009, dispôs sobre a Sistematização da Assistência de Enfermagem e, a implementação do Processo de Enfermagem em ambientes públicos ou privados, nos quais desenvolva-se o cuidado profissional de enfermagem. Em seu Art. 3, enfatiza que o Processo de Enfermagem deverá ter base teórica que o oriente nas etapas de: Coleta de Dados; Diagnóstico de Enfermagem; e, no Planejamento das Ações ou Intervenções de Enfermagem. Somente assim estará munido do (re) conhecimento das singularidades de cada indivíduo que está sob seus cuidados e, obterá subsídio para, a posteriori, avaliar os resultados alcançados com suas intervenções ${ }^{(3)}$.

Neste cenário, considerando as dificuldades vivenciadas por uma das autoras bem como, relatadas por enfermeiros de uma Unidade de Terapia Intensiva Pediátrica para o manejo da criança em suspeita de ME, aliada à proposta de um projeto de intervenção na realidade profissional no contexto do curso de pós-graduação profissional, é que emerge a produção de uma Tecnologia Cuidativo-Educacional(4).

Segundo Nietsche, Teixeira e Medeiros (2014)(4), as Tecnologias Cuidativo-Educacionais podem ser definidas pela potencialidade da sua aplicação no processo do planejamento, de cuidar e, de capacitar ou aperfeiçoar o profissional. Entende-se que as tecnologias educacionais tornam o processo de trabalho menos complexo e mediam o aprendizado como instrumento ou dispositivo utilizado no processo educador/educando.

Com estas considerações se destaca a relevância da presente apresentação que tem como objetivo relatar o método para produção de um protótipo para o manejo da criança em suspeita de morte encefálica, na modalidade de aplicativo-guia móvel.

\section{METODOLOGIA}

Como tipo de estudo adota-se o relato do método científico desenvolvido para obtenção da tecnologia. Neste sentido, adaptou-se os passos apontados por Echer5 para o desenvolvimento de tecnologia educacional, com a perspectiva de apresentar o percurso metodológico para a produção de uma Tecnologia Cuidativo-Educacional. O desenvolvimento se deu no período de janeiro a junho de 2019.

Será apresentada a seguinte sequência5 de produção da tecnologia: 1. Submissão ao Comitê de Ética e Pesquisa; 2. Busca na literatura técnico-científica e, normativa, para o conhecimento específico; 3. Triagem do conteúdo identificado; 4. Sondagem, junto aos participantes da pesquisa, da necessidade de desenvolvimento de tecnologia para o manejo da criança em suspeita de morte encefálica; 5. Elaboração de um formulário com conteúdo técnico-científico e, normativo, a ser utilizado para validação; 6. Qualificação da Tecnologia Cuidativo-Educacional; 7. Definição do lay-out; e, 8. Desenvolvimento de Aplicação Mobile.

O local do estudo foi a Unidade de Terapia Intensiva Pediátrica, do Complexo Hospital de Clínicas da Universidade Federal do Paraná - CHC/UFPR, no município de Curitiba. Quanto aos participantes da pesquisa, foram envolvidos profissionais enfermeiros e médicos de duas unidades do referido hospital público e de ensino, da Unidade de Terapia Intensiva Pediátrica e, da Comissão Intra-Hospitalar de Doação de Órgãos e Tecidos para Transplantes (CIHDOTT). E ainda, alguns profissionais da Central Estadual de Transplantes, da Secretaria de Saúde do Estado do Paraná CET/SESA/PR.

\section{RESULTADOS E DISCUSSÃO}

Após a definição de um Projeto congruente com a Sistematização da Assistência de Enfermagem e, com referencial teórico na Teoria das Necessidades Humanas Básicas, a trajetória metodológica para o desenvolvimento de uma Tecnologia Cuidativo-Educacional acompanhou passos sequenciais e sistematizados que serão explanados neste desenvolvimento. Os resultados são apresentados acoplados à discussão de nossa vivência durante este processo. 
PASSO 1. Submissão ao Comitê de Ética em Pesquisa em Seres Humanos

O Conselho Nacional de Saúde (CNS), um órgão consultor junto ao Ministério da Saúde e equipamentos do SUS, como também de controle social. Estetem com sua responsabilidade zelar pelos aspectos éticos das pesquisas em seres humanos: análise, acompanhamento, regulamentação, proteção dos participantes, recepção de análise de recursos por qualquer parte interessada, apreciação de propostas para áreas temáticas especiais - "que encerram dilemas éticos mais complexos". O mesmo recomendou em 1988, a criação da rede nacional de Comitês de Ética (CEP) nas instituições e, na Resolução 196, promulgada em 10 de outubro de 1996, que está vigente, constam as Diretrizes e Normas Regulamentadoras de Pesquisas envolvendo Seres Humanos ${ }^{(6)}$.

Assim, a presente pesquisa foi submetida ao Comitê de Ética em Pesquisa, em Seres Humanos do CHC/UFPR, município de Curitiba, com aprovação em 08 de outubro de 2018, sob o Parecer no 2.947.877.

O Projeto temático no qual se insere esta Pesquisa foi contemplado pelo Edital no 27/2016, do Acordo CAPES/ COFEN n 30/2016, apoio a Programas de Pós-graduação da Área de Enfermagem - Modalidade Mestrado Profissional. Mais especificamente, em seu Sub-projeto 1, que como objetivo geral apresentava: “Desenvolver tecnologias assistenciais que auxiliem o enfermeiro na avaliação clínica diária de pacientes hospitalizados". E, foram atendidos com o presente desenvolvimento, os objetivos específicos de "Desenvolver Tecnologia Assistencial para Avaliação do paciente pediátrico em morte encefálica" e "Implantar a tecnologia assistencial desenvolvida na unidade campo da pesquisa".

\section{PASSO 2: Busca na literatura técnico-cientifica e normativa}

Dentre as evidências em saúde produzidas, as mais atuais e fortes, ou seja, produzidas por estudos com desenhos confiáveis, devem ser aplicadas no desenvolvimento das práticas clínicas em enfermagem, direcionando a melhor qualidade do atendimento, com vistas à obtenção dos melhores resultados ${ }^{(7)}$. Pesquisadores alertam para o fato de que uma evidência isolada não é suficiente para sustentar uma prática e recomendam que se proceda à busca completa nas fontes e, na sequência, à sintese dos achados disponíveis e adequados ao que se pretende impactar ${ }^{(7)}$.

Sob esta ótica foi realizada a busca bibliográfica para obtenção do embasamento teórico do conhecimento específico, nas bases de dados: LILACS - Literatura Latinoamericana e do Caribe em Ciências da Saúde; Scientific Eletronic Library Online (SciELO) Citation Index (Web of Science); Serviço Nacional de Aprendizagem Comercial (SENAC) - Biblioteca Digital; Google Scholar; Web of Science
- Coleção Principal (Clarivate Analytics); Biblioteca Virtual em Saúde - BVS (BIREME) - Centro Latino-Americano e do Caribe de Informação em Ciências da Saúde. Para tanto aplicaramse os Descritores em Saúde (DECS), na lingua portuguesa, espanhola e inglesa, a saber: Morte Encefálica - Brain Death - Muerte Encefálica; Obtenção de Tecidos e Órgãos - Tissue and Organ Procurement - Obtención de Tejidos y Órganos; Tomada de Decisão - Decision Making - Toma de Decisiones; Cuidados Críticos - Critical Care-Cuidados Críticos; Cuidados de Enfermagem - Nursing Care - Atención de Enfermería; Avaliação em Enfermagem - Nursing Assessment - Evaluación en Enfermería; Processo de Enfermagem - Nursing Process - Proceso de Enfermería; Teoria de Enfermagem, - Nursing Theory - Teoría de Enfermería; Diagnóstico de Enfermagem Nursing Diagnosis - Diagnóstico de Enfermería; Planejamento - Planning - Planificación; Tecnologia - Technology Tecnología; Tecnologia Educacional - Educational Technology - Tecnología Educacional; Tecnologia Biomédica - Biomedical Technology - Tecnología Biomédica - utilizando-se variadas chaves de busca com arranjos mediados pelos operadores boleanos "AND" e "OR".

Para o arcabouço normativo foram acessadas Resoluções nacionais e estaduais, assim como, dos Conselhos Federais de Medicina e de Enfermagem. Obtiveram-se 21 documentos, entre leis, Resoluções e Portarias, a saber: Constituição Federal de 19888; a Lei no 9434/1997 que dispõe sobre a remoção de órgãos e tecidos para transplantes ${ }^{(9)}$; a Resolução do Conselho Federal de Enfermagem, COFEN no 292/2004, que normatiza a atuação do enfermeiro na doação e captação de órgãos e tecidos para transplantes10. Em 2017, a Portaria de Consolidação no 4, dispõem sobre a Consolidação das normas dos sistemas e subsistemas do Sistema Único de Saúde ${ }^{(11)}$ e, revoga: o Regulamento Técnico do Sistema Nacional de Transplante; o Decreto no 9174/2017(12), regulamentando a Lei $n$ o 9434/1997, que trata da disposição dos órgãos, tecidos, células e partes do corpo humano, para fins de transplante e tratamento. Assim, o Conselho Federal de Medicina atualiza os critérios de diagnóstico de morte encefálica com a Resolução CFM nㅇ $2173 / 2017^{(1)}$

\section{PASSO 3: Triagem do conteúdo identificado}

Com o resultado do processo de identificação das melhores evidências técnicas, científicas e normativas, associando-as ao conteúdo de livros-texto e, à prática profissional da pesquisadora mestranda profissional na área, bem como, ao conhecimento teórico e prático dos participantes da pesquisa, foram elencados os conteúdos e organizados sobre os seguintes tópicos teóricos que foram desenvolvidos nos subcapítulos denominados de Referencial conceitual-normativo (Doação de Órgãos e Tecidos; A 
Morte Encefálica) e, Revisão Teórico-empírica (Processo de Enfermagem; As Necessidades Humanas Básicas; Taxonomia de NADA-I; As Alterações Fisiopatológica e a Manutenção do Potencial Doador Pediátrico; Tecnologias em Saúde).

Destarte, foi realizada a síntese teórica e normativa que deverá nortear e determinar a assistência adequada e oportuna para o manejo da criança em suspeita de morte encefálica, de acordo com as alterações fisiopatológicas decorrentes do processo, e de sua complexidade.

Nesta sequência processual, foram selecionadas: as Necessidades Humanas Básicas, seus grupos e subgrupos; os Diagnósticos de Enfermagem, suas definições, características definidoras / fatores associados; e, as Intervenções de Enfermagem essenciais para manutenção da viabilização dos órgãos e tecidos a serem doados.

O conteúdo foi elaborado a partir do mapeamento cruzado entre os indicadores - elaborados à partir das necessidades humanas básicas relacionadas às alterações fisiopatológicas decorrentes da morte encefálica, bem como, das percebidas na avaliação clínica do enfermeiro e, os Diagnósticos de Enfermagem, segundo taxonomia de NANDA-I e Intervenções de enfermagem, para promoção da assistência de enfermagem segura, planejada e humanizada ao potencial doador e familiares, a qual refletirá positivamente na viabilidade captação e transplantação dos órgãos.

Ressalta-se queSchub etal. ${ }^{(7)}$ ao apresentarem uma revisão sobre a implementação da prática clínica de enfermagem com base em evidências, enfatizaram que, anteriormente à aplicação das evidências aos cuidados de enfermagem, há necessidade de se observar as preferências individualizadas dos pacientes e, relativizá-las considerando os seus atributos - idade, conhecimentos, expectativas, cultura, familiaridade com a tecnologia, dentre outros. $\mathrm{Na}$ abordagem em relato, estas considerações deverão ser partilhadas com os familiares da criança em suspeita de morte.

\section{PASSO 4: Legitimação da necessidade pelo desenvolvimento de tecnologia para o manejo da criança em suspeita de morte encefálica}

Esta etapa do método também pode ser inserida nos principios da Evidence-based Nursing Practice - EBNP considerando-a ser um processo de múltiplas etapas, que envolve o trabalho do enfermeiro clínico junto a sua equipe multiprofissional(7).

Destarte, os enfermeiros que atuam na Unidade de Terapia Intensiva Pediátrica foram solicitados a participar, mediante esclarecimento do objetivo, leitura e assinatura do Termo de Consentimento Livre e Esclarecido (TCLE). Realizou-se a sondagem do conhecimento teórico aplicado na prática de avaliação clínica do paciente sob seus cuidados, bem como, ao manejo dos pacientes pediátricos em suspeita de morte encefálica.

Para tanto, foi desenvolvido um formulário semiestruturado no Google-docs e encaminhado via What'sApp. Mediante análise simples das respostas identificou-se que significativo número de enfermeiros apontou para a necessidade de maior conhecimento para o manejo e manutenção do potencial doador pediátrico. Este achado convergiu com o pressuposto que norteou o desenvolvimento da pesquisa de que uma tecnologia que apoiasse o enfermeiro para o manejo do potencial doador, seria oportuna para propiciar o desenvolvimento do Processo de Enfermagem, sobretudo para o Plano de Cuidados.

\section{PASSO 5. Construção de Formulário com conteúdo técnico- científico e, normativo, para validação}

Segundo Galdeano(14) validar significa o ato ou efeito de tornar algo válido, legítimo, isto é, tornar algo verdadeiro, algo cuja autenticidade é comprovada. A partir da definição do conteúdo teórico-científico e empírico, optou-se por dividir o cuidado à criança em suspeita de morte encefálica, em quatro Blocos, de tal forma, que contemplassem as fases do protocolo de morte encefálica, definidas pelo CFM, na Resolução no2173/2017 e, foi criado e denominado de "Formulário para Validação de Conteúdo da Tecnologia Cuidativo-educacional para manutenção do potencial doador pediátrico".

Neste Formulário, cada Bloco foi apresentado em um quadro, com oito colunas identificadas como: Número de Ordem; Diagnóstico de Enfermagem; Intervenções de Enfermagem / Procedimentos; Justificativa; seguidas pelas colunas para opções de concordância ou não, com registros de ' $X$ ' para positividade, com a possibilidade de sinalizarem por sua adequabilidade, sendo aplicada a letra 'A', para quando considerado adequado, 'PA' para parcialmente adequado e, 'I' para inadequado. Assim, foi utilizado o modelo da Escala de Likert, o qual possibilita ao participante, expor o grau de concordância a respeito de uma afirmação(15). Como última coluna havia a abertura para as sugestões para ajustes, que deveria ser preenchida pelos respondentes com sinalizações de itens 'PA' ou 'I' para a intervenção(15) (FIGURA l).

FIGURA 1: Cabeçalho do Formulário para Validação de Conteúdo da Tecnologia Cuidativo-educacional para manutenção do potencial doador pediátrico, Curitiba, Paraná, 2019

Diagnístico de Enfermagemt Procedimentos Intervençōes de Justificativa A PA Ajustes sugeridos em caso de Procedine 
Como juizes participantes foram selecionados os profissionais com expertise na temática e que mantinham proximidade da prática profissional de manejo do potencial doador. Os mesmos receberam uma Carta-convite explicativa e, assinaram ao TCLE. O Formulário foi entregue individualmente em mãos, em formato impresso e, foi solicitado que, analisassem o material com base no critério de adequação. O prazo solicitado para devolução do instrumento foi de sete dias, o mesmo foi cumprido pelos avaliadores, alguns deles além de entregar o formulário, solicitaram um período para exposição verbal de suas colocações e valorização do protótipo.

\section{PASSO 6. Qualificação da Tecnologia Cuidativo-Educacional} mediante análise do conteúdo

Realizado, pela pesquisadora, a análise das contribuições para o aprimoramento da tecnologia, utilizando o IVC Índice de Validade do Conteúdo(10), para avaliar o grau de concordância e discordância do Conteúdo da Tecnologia, pelos profissionais juízes. Este mensura a proporção da concordância dos experts sobre cada item exposto para análise, e seu cálculo é feito com a somatória das respostas 'A' (Adequada) dividida pelo número total das respostas em cada uma das alternativas, conforme equação: IVC = № de respostas $A / N$ № total de respostas.

A Taxa de Concordância aceitável entre os juízes para avaliação dos itens individualmente deve ser superior a 0,7840 segundo os autores ${ }^{(10)}$, e foi adotada nesta pesquisa. Os itens com IVC inferiores a 0,7840 foram readequados conforme as sugestões dos juízes/experts ${ }^{(16,17)}$. O resultado da avaliação, foi organizado em outro quadro, agregando-se os itens do conteúdo validado, o IVC e, discriminando-se em redação, os ajustes implementados que atenderam às sugestões dos juízes. Ressalta-se que nesta etapa, com vistas à uma melhor organização visual, e atendendo às solicitações de alguns dos juízes, a disposição no Quadro foi realizada aproximando-se os Procedimentos e, na mesma lógica, os Diagnósticos de Enfermagem. Sob a mesma ótica foi organizado o Bloco I em formato de checklist a ser checado anteriormente à abertura do Protocolo. Os demais blocos mantiveram a estrutura previamente proposta.

\section{PASSO 7. Definição do layout do protótipo}

O layout foi definido a partir da estrutura gráfica, utilizando o software adobe $X D^{(18)}$ - ferramenta baseada em vetores, para projetar e criar protótipos de experiência do usuário para aplicativos da Web e móveis (ADOBE, 2018)(18). Esta fase da tecnologia foi desenvolvida pela Associação Júnior de Desenho Industrial "Junior Design", da Universidade Federal do Paraná (UFPR).
A estrutura gráfica ou mapa conceitual, pode ser compreendida como a representação visual utilizada para compartilhar as definições, considerando que esclarece a maneira que o autor explicita o elo entre os conceitos expostos. Estes, são considerados mais práticos para a transmissão das informações quando comparados aos textos, devido a facilidade de sua acessibilidade e usabilidade ${ }^{(19)}$.

\section{PASSO 8. Desenvolvimento de Aplicação Mobile}

Utilizou-se a metodologia ágil de Scrum, aplicada na gestão e planejamento de projetos de softwares. É tida como extremamente ágil e flexível, e se baseia no desenvolvimento incremental das aplicações, centrado na equipe, com ciclos de interação curtos ${ }^{(20)}$. Foi desenvolvido pela Associação Junior de Consultoria em Informática "Ecomp", da Universidade Federal do Paraná (UFPR), nos padrões atuais requeridos pela Google Play e App Store.

\section{Limitações do Estudo:}

Reitera-se que por se tratar de uma tecnologia envolvendo áreas de designer e informática a produção de aplicação mobile na área de saúde requer a busca e, o aprimoramento do conhecimento específico, com demanda por tempo de dedicação mais prolongado.

\section{Contribuições do estudo para a prática:}

Esta tecnologia é inovadora e subsidiará o enfermeiro na identificação das alterações fisiopatológicas, no desenvolvimento do Processo de Enfermagem e adoção das condutas oportunas para o manejo da criança em suspeita de morte encefálica e do potencial doador pediátrico, de modo a manter viáveis os órgãos e tecidos para transplantação. Contribuirá também, para a educação continuada, qualificando e inovando o processo de cuidado para a manutenção do potencial doador pediátrico, com potencial cobertura institucional, regional, estadual e, quiçá, nacional. Considera-se este produto como reprodutivel para outros segmentos etários, e, a ele se reserva um impacto social que privilegia a solidariedade, sendo uma tecnologia que prevê impacto social na área do transplante pediátrico.

\section{CONCLUSÃO}

Por fim, admite-se como relevante o presente relato sistematizado de método para a construção de uma tecnologia Cuidativo-Educacional, modalidade aplicação mobile, para orientar os enfermeiros no desenvolvimento de outras tecnologias aplicáveis para qualificação de sua prática em cenário específico.

Compreender, analisar e seguir o processo descrito neste estudo pode ser essencial para os pesquisadores e os 
profissionais da área de saúde que estiverem preocupados em produzir e aplicar instrumentos confiáveis e, apropriados às suas práticas.

\section{Contribuições dos autores}

Concepção e/ou desenho: Tolari VA, Freire, MHS; Análise e interpretação dos dados: Tolari VA, Freire, MHS; Redação do artigo: Tolari VA, Freire, MHS; Revisão crítica: Tolari VA, Freire, MHS; Revisão final: Tolari VA, Freire, MHS.

\section{Agradecimentos:}

Agradecemos ao Conselho Federal de Enfermagem (COFEN) e a Coordenação de Aperfeiçoamento de Pessoal de Nivel Superior (CAPES), pelo acordo COFEN/CAPES, Edital 27/2016, que proporcionou financiamento para a produção desta Tecnologia Cuidativo-Educacional e, ao Programa de Pós-graduação em Enfermagem Profissional da Universidade Federal do Paraná por disponibilizar a oportunidade de ingresso.

\section{REFERÊNCIAS}

1. Conselho Federal de Medicina. Resolução no 2173/2017, de 15 de dezembro de 2017. Define os Critérios de morte encefálica. Acesso em 10/04/18. Disponivel em: http://www.saude.rs.gov.br/upload/ arquivos/carga20171205/19140504-resolucao-do-conselhofederal-de-medicina-2173-2017.pdf

2. Secretaria Estadual de Saúde de Curitiba. Sistema Estadual de Transplantes do Paraná. Manual para Notificação, Diagnóstico de Morte Encefálica e Manutenção do Potencial Doador de Órgãos e Tecidos. - Curitiba: SESA/SGS/CET, 2018. 62p. Pag.30-5.

3. Conselho Federal de Enfermagem. Resolução no 358, de 15 de outubro de 2009. Dispõe sobre a Sistematização da Assistência de Enfermagem e a implementação do Processo de Enfermagem em ambientes, públicos ou privados, em que ocorre o cuidado profissional de enfermagem, e dá outras providências. Acesso em 15/04/2018. Disponivel em: http://www.cofen.gov.br/resoluocofen-3582009_4384.html

4. Nietsche EA, Teixeira E, Medeiros HP. Tecnologias Cuidativoeducacionais: Uma possibilidade de empoderamento do/a enfermeiro/a?. 1a edição. Porto Alegre. Moriá. 2014.208p. Pag. 11350.

5. Echer IC. Elaboração de manuais de orientação para o cuidado em saúde. Revista Latino-Americana de Enfermagem. 2005. Acesso em: 10/06/2019. vol. 13. núm. 5. pp. 754-757. Disponivel em: https:// www.redalyc.org/pdf/2814/281421849022.pdf

6. Ministério da Saúde (BR). Conselho Nacional de Saúde. Resolução CNS 196/96. Diretrizes e Normas Regulamentadoras de Pesquisas envolvendo Seres Humanos. Acesso em 10/11/2018. Disponivel em: https://conselho.saude.gov.br/comissao/conep/resolucao.html

7. Schub E, Walsh K, Pravikoff D.Evidence-Based Nursing Practice: Implementing. 2017

Nursing Reference Center. Pag.1-18. Acesso em 20/05.2019. Disponivel em: http://web.a.ebscohost.com/nup/delivery?sid=fce 4 f12d-04ac- 42 ... d\%3dfce4f12d-04ac-4203-b614-48b54155a281\%2540sessionmgr4006

8. Brasil. Constituição de 1988, de 05 de outubro de 1988. Constituição da república Federativa do Brasil, Acesso em 10/04/2018. Disponivel em:

https://www2.camara.leg.br/legin/fed/consti/1988/constituicao1988-5-outubro-1988-322142-publicacaooriginal-1-pl.html

9. Brasil. Lei no 9434, de 04 de fevereiro de 1997. Dispões sobre a remoção de órgãos, tecidos e partes do corpo humano para fins de transplantes e tratamento e dá outras providências. Acesso em 10/04/18. Disponivel em: http://www.saude.pr.gov.br/arquivos/File/ centraldetransplantes/Lei9434.pdf

10. Conselho Federal De Enfermagem. Resolução COFEN no 292 de Junho de 2004. Normatiza a atuação do Enfermeiro na Captação e Transplante de Órgãos e Tecidos. Acesso em 20/10/2018. Disponivel em : http://www.cofen.gov.br/resoluo-cofen-2922004_4328.html

11. Ministério da Saúde (BR) Gabinete do Ministro. Portaria de Consolidação nㅇ 5, de 28 de setembro de 2017. Consolidação das normas sobre as ações e os serviços de saúde do Sistema Único de Saúde. Acesso em 09/11/2018 Disponivel em: http://bvsms.saude. gov.br/bvs/saudelegis/gm/2017/prc0005_03_10_2017.html

12. Casa Civil (BR). Decreto $\mathrm{n} \cong 9175$ de 18 de outubro de 2017. Regulamenta a Lei no 9.434, de 4 de fevereiro de 1997, para tratar da disposição de órgãos, tecidos, células e partes do corpo humano para fins de transplante e tratamento. Acesso em 10/04/2018. Disponivel em: http://www.planalto.gov.br/ccivil_03/_Ato20152018/2017/Decreto/D9175.htm

13. NANDA-I. North American Nursing Diagnosis Association International. Diagnósticos de enfermagem da NANDA: definições e classificação - 2018-2020. Porto Alegre: Artmed, 2018. 606 p. Acesso em 10/11/2018. Disponivel para uploads em: http://nascecme.com. br/2014/wp-content/uploads/2018/08/NANDA-I-2018_2020.pdf

14. Galdeano LE. Rossi LA, Pelegrino FM. Validação de conteúdo do diagnóstico de enfermagem conhecimento deficiente. 2008. Acesso em 19/03/2019.Acta paul. Enferm. Vol.21 no.4. Disponivel em: http://www.scielo.br/scielo.php?script=sci_ arttextEpid=S0103-21002008000400003

15. Silva Junior SD, Costa FJ. Mensuração e escalas de verificação: uma Análise Comparativa das Escalas de Likert e Phrase Completion. 2014. Acesso em: 31/05/2019. Revista Brasileira de Pesquisas de Marketing, Opinião e Midia ISSN: 1983-9456 (Impressa) ISSN: 2317-0123 (On-line).Disponivel em: http://sistema.semead.com. br/17semead/resultado/trabalhosPDF/1012.pdf

16. Polit DF, Beck CT. Fundamentos da pesquisa em enfermagem: avaliação de evidênciaspara a prática de enfermagem. 7ạ ed. Porto Alegre: ArtMed; 2011. Pag.150-3.

17. Alexandre NMC, Colucci MZO. Validade de conteúdo nos processos de construção e adaptação de instrumentos de medidas. 2011. Acesso em 10/02/2019 vol.16 no.7 Rio de Janeiro Disponivel em : http://www.scielo.br/scielo.php?script=sci_ arttextEpid=S1413-81232011000800006

18. ADOBE "Adobe XD", 2018 Acesso em: junho de 2019.Disponivel em : https://www.adobe.com/br/products/xd.html.

19. Tavares R. Constructing concept maps. 2007.Acesso em 31/05/2019. vol.12, pp. 72-85. Disponivel em: http://pepsic.bvsalud. org/scielo. php?pid=S1806-58212007000300008\&script=sci_ abstractEtlng=en

20. Bissi W. SCRUM - Metodologia de desenvolvimento ágil. 2007. Acesso 31/05/2019. Campo Mourão. v.2. n.1,p.3-6. Campo Dig. .Disponivel em: https://scholar.google.com.br/scholar?hl=pt-BREas_ sdt $=0 \% 2 \mathrm{C} 5$ \& q $=U \mathrm{~m}+$ Modelo+\%C3\%8lgil +para+Gest $\%$ C3\%A3o+de+Projetos+de+Software. EbtnG $=$ 